\title{
Management of pain after Cesarean delivery without intrathecal morphine: networking for the best answer
}

\author{
Garrett Barry, MD · Vishal Uppal, MBBS, MSc, FRCA $\unrhd$
}

Received: 21 August 2020/Revised: 21 August 2020/Published online: 6 October 2020

(C) Canadian Anesthesiologists' Society 2020

Acute pain after Cesarean delivery (CD) is one of the most important patient-centred outcomes that an obstetric anesthesiologist can influence. Severe acute pain after $\mathrm{CD}$ results in patient dissatisfaction, impaired recovery, longer hospital stay, and delayed return to normal activities. It has also been shown to predict long term adverse outcomes such as a higher rate of persistent postsurgical pain and post-partum depression at eight weeks. ${ }^{1}$

Since the majority of $\mathrm{CDs}$ are performed under neuraxial anesthesia, the use of intrathecal opioids is a common and convenient way of providing analgesia following $\mathrm{CD}$ along with a multimodal analgesic regimen. Nevertheless, in scenarios where either spinal anesthesia or intrathecal morphine (ITM) are contraindicated or best avoided, alternatives to ITM are required. The alternatives include single-injection or continuous local anesthetic wound infiltration, or truncal blocks such as the transversus abdominis plane (TAP) block or quadratus lumborum block (QLB). A recent review of post-CD regional analgesia techniques discusses each of these approaches in detail. ${ }^{2}$

In this issue of the Journal, Sultan et al. present a network meta-analysis (NMA) comparing single-shot TAP block, single-dose wound infiltration (WI), and continuous wound catheter (WC) with a primary outcome of 24-hr opioid consumption after $\mathrm{CD} .^{3}$ At first glance, it may be unclear from this study which technique is superior. Ultimately, TAP block finds the optimal balance of analgesic efficacy and feasibility. The analysis included

G. Barry, MD · V. Uppal, MBBS, MSc, FRCA ( $₫)$

Department of Anesthesia, Perioperative Medicine and Pain Management, Nova Scotia Health Authority and IWK Health Centre, Dalhousie University, Halifax, NS B3H 2Y9, Canada e-mail:v.uppal@dal.ca
42 studies comprising 2,906 patients who did not receive ITM or alternative long-acting intrathecal opioids. The TAP block and WC showed lower 24-hr opioid consumption when compared with inactive controls. Nevertheless, there was no difference between WI and inactive control for this outcome. Further, both TAP blocks and WC showed lower 12-hr (NRS) pain scores at rest and movement compared with WI and inactive controls. Similarly, the NRS 24-hr rest pain scores were lower for the TAP and WC groups compared with the inactive controls. Finally, the time-to-first analgesic request was also longer for the TAP block than inactive controls. There were no statistically significant differences in the remaining secondary outcomes, including 24-hr movement NRS pain scores, postoperative nausea and vomiting, maternal sedation, and pruritis.

This systematic review found that most randomizedcontrolled trials (RCTs) compared single-injection TAP blocks or WI techniques with an inactive control while only a few directly compared TAP block with WC or WI. There were no RCTs comparing WC with WI. In this situation, where the comparisons of interest were not studied directly, the NMA can be a useful methodology to analyze a network of three or more interventions in a set of studies by combining direct and indirect comparisons to estimate the effects of each treatment. ${ }^{4}$

Direct estimates of treatment effect can be calculated when trials have compared two different treatments, such as treatment A vs B and treatment A vs C (Figure a, solid lines). Network meta-analysis also calculates estimates of the effect from the network when no direct comparisons are available, but the trials share a common comparator. These are called indirect estimates: for example, treatment B $v s$ $\mathrm{C}$, as shown by the dashed line in Figure a. The validity of NMA relies on satisfying the assumption of transitivity: 


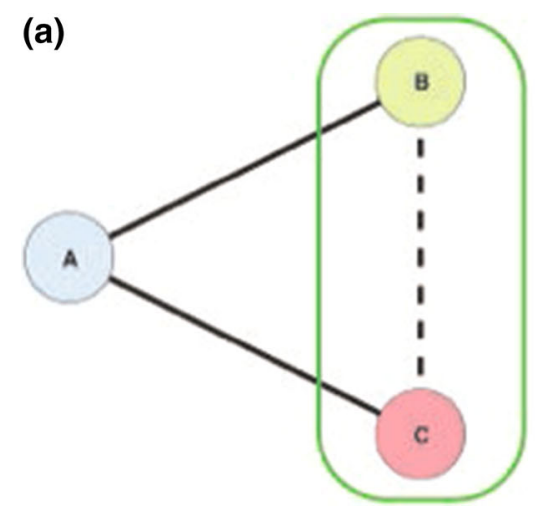

Figure a) There are direct comparisons (solid lines) between treatment $\mathrm{A}$ and $\mathrm{B}$, treatment $\mathrm{A}$ and $\mathrm{C}$, but not treatment $\mathrm{B}$ and $\mathrm{C}$. Treatment $\mathrm{B}$ and $\mathrm{C}$ share the common comparator treatment $\mathrm{A}$, so indirect effect estimates are made (dashed line). b) When direct comparisons between treatment $\mathrm{B}$ and treatment $\mathrm{C}$ are also available, network meta-analysis calculates effect estimates using both direct and indirect comparisons.a) Il y a des comparaisons directes (lignes

that there are no systematic differences between patients across trials such that they were equally likely to have been randomized to either treatment. When both direct and indirect estimates are available (Figure b), the NMA calculates mixed estimates of the treatment effect. If the direct and indirect estimates are consistent with each other, confidence in the findings is strengthened. On the other hand, if the direct and indirect estimates suggest differing treatment decisions, there is less confidence in the results, and the certainty of evidence may be subsequently downgraded.

The effect sizes in this NMA are reported as standardized mean differences (SMDs), which provide an accurate comparison of treatment groups across studies using disparate outcome scales, but can be challenging to interpret clinically since the cut-off set for these effect sizes are relatively arbitrary and are numerical (rather than clinical) in nature. Standardized mean differences do not readily translate to a familiar clinical value, such as the absolute difference in morphine equivalents between treatment groups. Nevertheless, the NMA also generates a ranking (surface under the cumulative ranking curve [SUCRA]) of the treatments in order of likelihood of producing the best outcome. Given the difficulty of interpreting SMDs in this study, the network ranking provides a clinically useful recommendation in favour of TAP blocks and WC over WI and inactive controls.

While single-shot TAP block and WC techniques appear almost comparable in efficacy, the greater feasibility of the TAP block gives it an edge. Catheter-based techniques are undesirable in the obstetric population for several key reasons. First, the presence of a catheter and associated pump impairs early patient mobility, requires extra time and training for staff, and likely increases the overall cost

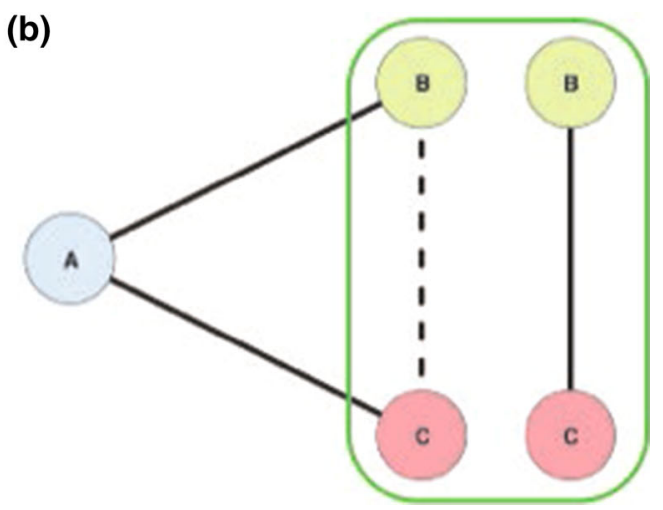

continues) entre les traitements $\mathrm{A}$ et $\mathrm{B}$, les traitements $\mathrm{A}$ et $\mathrm{C}$, mais pas entre les traitements B et C. Les traitements B et $\mathrm{C}$ partagent le traitement A comme comparateur commun, alors des estimations d'effet indirectes sont calculées (ligne tiretée). b) Lorsque des comparaisons directes entre le traitement $\mathrm{B}$ et le traitement $\mathrm{C}$ sont aussi disponibles, la méta-analyse en réseau calcule les estimations d'effet à l'aide de comparaisons directes et indirectes.

of care. Generally speaking, obstetric patients are relatively healthy and able to recover from surgery quickly; iatrogenic barriers to a quick recovery are contradictory to the tenets of "enhanced recovery after CD" and should be avoided in the absence of substantial evidence. ${ }^{5}$ Second, the risk of catheter dislodgement during activity essentially leads to the failure of the analgesic technique. Third, the use of a catheter, whether in a wound site or fascial plane, exposes the patient to a higher risk of local anesthetic systemic toxicity, which is particularly problematic in low acuity post-partum units where adequate monitoring and training to deal with this feared complication may be lacking. Finally, WCs may increase the risk of wound infection.

Transversus abdominis plane blocks involve the deposition of the local anesthetic in the fascial plane between the transversus abdominis and internal oblique muscles. ${ }^{6}$ The exact location of the injection is quite important. Posterior TAP injections have been shown to be superior to lateral TAP blocks in terms of 24-hr opioid consumption and pain scores. ${ }^{7}$ This may be due to the more complete coverage of the thoracoabdominal nerves before they branch laterally in the TAP plane. The potential benefits of the TAP block may have been diluted in the NMA by Sultan et al. since lateral and posterior TAP blocks were grouped together. The main limitations of the TAP block are that it needs provider expertise, and there is a small risk of local anesthetic systemic toxicity. While most surgeons are comfortable with WI, this may not be the case with respect to TAP blocks and thus may require the expertise of the anesthesiologist. With respect to the risk of local anesthetic toxicity, ultrasound guidance may mitigate the risk of inadvertent intravascular injection compared with the landmark technique. ${ }^{8}$ 
The comparison of TAP catheters with WCs would have been of interest to clinicians, but no obstetric studies used TAP catheters. The dearth of TAP catheter studies in the obstetric population is not surprising given additional feasibility and safety problems as already mentioned regarding WC. ${ }^{6}$ Whether there is any analgesic superiority of TAP catheters to single-shot TAP blocks remains uncertain in the $\mathrm{CD}$ population. Nevertheless, a recent study showed no benefit of TAP catheters over single-shot TAP blocks in patients having laparoscopic live-donor nephrectomy. ${ }^{9}$ Catheter techniques, including postoperative epidural analgesia, may have their place in certain patient populations where pain management is likely to be challenging, such as highly opioid-tolerant patients and patients taking opioid agonist-antagonist therapies.

The QLB was absent from the study by Sultan et al. despite promising results from recent RCTs. A recent metaanalysis concluded that the QLB significantly reduced 24$\mathrm{hr}$ opioid consumption when compared with inactive controls in the absence of ITM after CD. ${ }^{10}$ Quadratus lumborum block is essentially an extension of the posterior TAP block; local anesthetic is deposited in the thoracolumbar fascial planes adjacent to the quadratus lumborum muscle. With QLB, the injectate may spread to the paravertebral space, blocking nerve fibres supplying the abdominal viscera. ${ }^{6,11}$ A recent NMA comparing QLB with TAP blocks in the Cesarean population found only two small RCTs directly comparing TAP and QLB. Both RCTs favoured QLB in terms of reducing morphine consumption and prolonging time-to-first analgesic request. Nevertheless, combining direct and indirect estimates, there was insufficient evidence to firmly conclude whether one technique was superior to the other with respect to postoperative 24-hr opioid consumption and pain scores. ${ }^{12}$ Both TAP and QLB blocks are associated with significantly lower postoperative opioid consumption in the absence of ITM compared with control.

Beyond the particular regional technique, the benefits of multimodal analgesia and adjuvants to regional analgesia should not be forgotten in the obstetric context. The wellknown opioid-sparing qualities of scheduled non-steroidal anti-inflammatory drugs (NSAID) and acetaminophen are foundational to any analgesia strategy. ${ }^{13}$ Dexamethasone can be considered as a part of a multimodal analgesic regimen. A single intravenous dose of dexamethasone has been shown to reduce postoperative pain, ${ }^{14}$ prolong the nerve block duration, ${ }^{15}$ and provide an antiemetic effect. While routine during general anesthesia, intravenous dexamethasone is much less commonly administered to patients having regional anesthesia despite its analgesic and antiemetic benefits.
For post-CD analgesia, the current state of the evidence suggests the use of aggressive multimodal analgesia with scheduled acetaminophen and NSAIDs plus a single-shot ultrasound-guided posterior TAP block in the absence of long-acting intrathecal opioid. This regimen strikes the optimal balance of efficacy, feasibility, and safety. Intravenous dexamethasone can be considered a safe and effective adjunct to prolong block analgesia after CD. A single-dose WI has no proven benefit over an inactive control. The continuous WC techniques are unlikely to confer enough analgesic benefit over TAP blocks to warrant their use, given the hindrances to patient recovery. Recent evidence suggests the QLB is at least equivalent to TAP blocks. Given promising results in favour of QLB in head-to-head trials, further studies comparing QLB with TAP block are warranted.

\section{Prise en charge de la douleur après un accouchement par césarienne sans morphine intrathécale : réseautage pour la meilleure réponse}

La douleur aiguë après un accouchement par césarienne est l'un des plus importants devenirs centrés sur la patiente sur lequel un anesthésiologiste obstétrical peut avoir un impact. Une importante douleur aiguë post-césarienne entraîne l'insatisfaction de la patiente, un rétablissement plus laborieux, une durée de séjour hospitalier prolongée, et un retour retardé aux activités normales. Il a également été démontré que ce type de douleur était un prédicteur de devenirs défavorables à long terme, tels que des taux plus élevés de douleur post-chirurgicale persistante et de dépression postpartum à huit semaines. ${ }^{1}$

Étant donné que la majorité des accouchements par césarienne sont réalisés sous anesthésie neuraxiale, l'utilisation d'opioïdes neuraxiaux est une approche aussi répandue que pratique pour procurer une analgésie à la suite d'une césarienne, lorsque ces agents sont administrés de concert avec un régime analgésique multimodal. Toutefois, dans les cas où une rachianesthésie ou une administration de morphine intrathécale (MIT) sont contreindiquées, ou s'il est préférable d'éviter ces modalités, des alternatives à la MIT sont alors nécessaires. Les alternatives comprennent l'infiltration de l'incision avec un anesthésique local en injection unique ou en perfusion 
continue, ou les blocs du tronc tels que le bloc du muscle transverse de l'abdomen (bloc TAP - Transversus Abdominis Plane) ou le bloc du muscle carré des lombes (quadratus lumborum). Un article de revue récent s'intéressant aux techniques d'analgésie régionale postcésarienne décrit chacune de ces approches en détail. ${ }^{2}$

Dans ce numéro du Journal, Sultan et coll. présente une méta-analyse en réseau (MAR) comparant les blocs TAP à injection unique, les infiltrations de l'incision en dose unique, et les infiltrations continues via cathéter. Le critère d'évaluation principal était la consommation d'opioïdes cumulative à $24 \mathrm{~h}$ après un accouchement par césarienne. ${ }^{3}$ À première vue, il pourrait sembler difficile de déterminer quelle technique est supérieure. En bout de ligne, le bloc TAP permet d'atteindre l'équilibre optimal entre efficacité analgésique et faisabilité. L'analyse a inclus 42 études comportant un total de 2906 patientes n'ayant pas reçu de MIT ou d'autres opioïdes intrathécaux à action prolongée. Le bloc TAP et l'infiltration en perfusion continue ont entraîné une consommation d'opioïdes sur $24 \mathrm{~h}$ plus faible par rapport aux comparateurs inactifs. Aucune différence n'a cependant été observée entre l'infiltration en dose unique et les comparateurs inactifs pour ce critère d'évaluation. En outre, les blocs TAP et les infiltrations en continu ont été associés à des scores de douleur (EVA) plus bas à $12 \mathrm{~h}$ au repos et en mouvement par rapport aux infiltrations à dose unique et aux comparateurs inactifs. De la même manière, les scores de douleur sur l'EVA à $24 \mathrm{~h}$ au repos étaient plus bas dans les groupes bloc TAP et infiltration continue que dans les groupes comparateurs inactifs. Enfin, le délai jusqu'à la première requête d'analgésiques était également plus long dans le groupe bloc TAP qu'avec les comparateurs inactifs. Aucune différence statistiquement significative n'a été observée dans les critères d'évaluation secondaires restants, y compris dans les scores de douleur sur l'EVA à $24 \mathrm{~h}$ en mouvement, ou en matière de nausées et vomissements postopératoires, de sédation maternelle, et de prurit.

Cette revue systématique révèle que la plupart des études randomisées contrôlées (ERC) réalisées ont comparé des techniques de blocs TAP et d'infiltrations uniques à un comparateur inactif, alors que seules quelques-unes comparaient directement les blocs TAP aux infiltrations uniques ou continues. Aucune ERC n'a comparé les infiltrations en continu aux infiltrations à dose unique. Dans cette situation, lorsque les comparaisons qui intéressent les auteurs ne sont pas directement étudiées, une MAR peut constituer une méthodologie utile pour analyser un réseau de trois interventions ou plus dans un ensemble d'études, en combinant des comparaisons directes et indirectes afin d'estimer les effets de chaque traitement. ${ }^{4}$

Les estimations directes de l'effet d'un traitement peuvent être calculées lorsque les études ont comparé deux traitements différents, comme par exemple un traitement A $v s$ B et un traitement A vs C (figure a, lignes continues). La méta-analyse en réseau calcule également des estimations de l'effet à partir du réseau lorsqu'aucune comparaison directe n'est disponible, mais que les études partagent un comparateur identique. On parle alors d'estimations indirectes : par exemple, le traitement B vs C, comme illustré par la ligne en tireté de la figure a. La validité d'une MAR s'appuie sur l'hypothèse de transitivité, soit qu'il n'existe pas de différence systématique entre les patients d'une étude à l'autre, de telle sorte que les patients auraient tout aussi bien pu être randomisés à recevoir un traitement ou l'autre. Lorsque les estimations directes et indirectes sont disponibles (figure b), la MAR permet de calculer des estimations mixtes de l'effet du traitement. Si les estimations directes et indirectes sont cohérentes les unes par rapport aux autres, la confiance dans les résultats en est alors renforcée. En revanche, si les estimations directes et indirectes font apparaître des décisions de traitement divergentes, les résultats sont par conséquent moins fiables et la qualité des données probantes pourrait donc être subséquemment rétrogradée.

Les tailles d'effet de cette MAR sont rapportées en tant que différences moyennes standardisées (DMS), lesquelles fournissent une comparaison exacte de groupes de traitement dans des études utilisant des échelles d'évaluation disparates. Ces résultats peuvent toutefois être difficiles à interpréter d'un point de vue clinique, car les limites établies pour ces tailles d'échantillon sont relativement arbitraires et sont de nature numérique plutôt que clinique. Les différences moyennes standardisées ne sont pas facilement convertibles en valeurs cliniques familières, telle que la différence absolue en équivalents de morphine entre des groupes de traitement. Toutefois, la MAR génère également un classement (aire sous la courbe de rang cumulée [SUCRA]) des traitements dans leur ordre de 'probabilité de produire le meilleur résultat clinique'. Étant donné la difficulté d'interprétation des DMS dans cette étude, la classification du réseau propose une recommandation utile d'un point de vue clinique en faveur des blocs TAP et des infiltrations par perfusion continue, par rapport aux infiltrations uniques et aux comparateurs inactifs.

Alors que les techniques de blocs TAP en injection unique et d'infiltrations continues semblent quasiment comparables en matière d'efficacité, la plus grande faisabilité du bloc TAP lui donne un avantage. Les techniques par cathéter ne sont pas souhaitables dans la population obstétricale pour de multiples raisons. Premièrement, la présence d'un cathéter et de la pompe qui y est attachée entrave la mobilité précoce de la patiente, nécessite davantage de temps et de formation pour le 
personnel, et augmente probablement le coût global des soins. En règle générale, les patientes obstétricales sont en relativement bonne santé et capables de se remettre d'une chirurgie rapidement; les obstacles iatrogéniques à un prompt rétablissement sont contraires aux principes de la « récupération améliorée après un accouchement par césarienne " et devraient être évités en l'absence de données probantes concluantes. ${ }^{5}$ Deuxièmement, le risque de déplacement du cathéter pendant l'activité mène essentiellement à l'échec de la technique analgésique. Troisièmement, l'utilisation d'un cathéter, que ce soit au site d'incision ou dans un plan fascial, expose la patiente à un risque plus élevé de toxicité systémique de l'anesthésique local, ce qui est particulièrement problématique dans les services postpartum, services dans lesquels le monitorage et la formation adaptés pour prendre en charge cette complication redoutée pourraient faire défaut. Enfin, les infiltrations en perfusion continue pourraient augmenter le risque d'infection de l'incision.

Les blocs TAP sont réalisés en déposant l'anesthésique local dans le plan fascial, entre le muscle transverse de l'abdomen et le muscle oblique interne. ${ }^{6}$ L'endroit exact de l'injection est crucial. Il a été démontré que les injections postérieures de blocs TAP étaient supérieures aux blocs TAP latéraux en termes de consommation d'opioïdes et de scores de douleur à $24 \mathrm{~h}^{7}$ Cela pourrait être dû à la couverture plus exhaustive des nerfs thoraco-abdominaux avant qu'ils ne bifurquent latéralement dans le plan du muscle transverse de l'abdomen. Les avantages potentiels du bloc TAP pourraient avoir été dilués dans la MAR de Sultan et coll. étant donné que les blocs TAP latéraux et postérieurs étaient regroupés dans une même catégorie. Les limites principales des blocs TAP sont qu'ils nécessitent un clinicien expérimenté, et qu'il existe un faible risque de toxicité systémique de l'anesthésique local. Alors que la plupart des chirurgiens sont à l'aise avec les infiltrations uniques, cela pourrait ne pas être le cas en matière de blocs TAP et nécessiterait donc l'expertise de l'anesthésiologiste. En ce qui touche au risque de toxicité de l'anesthésique local, l'échoguidage pourrait mitiger le risque d'injection intravasculaire accidentelle par rapport à une technique fondée sur les repères anatomiques. ${ }^{8}$

La comparaison entre cathéters pour blocs TAP et infiltrations continues aurait été intéressante pour les cliniciens, mais aucune étude obstétricale n'a utilisé de cathéter avec des blocs TAP. L'absence d'études avec des cathéters pour les blocs TAP dans la population obstétricale est peu surprenante étant donné les problèmes supplémentaires de faisabilité et de sécurité, comme nous l'avons déjà mentionné en ce qui touchait aux infiltrations en perfusion continue. ${ }^{6}$ Dès lors, il est impossible de déterminer avec certitude si un bloc TAP par cathéter serait supérieur, d'un point de vue analgésique, à un bloc TAP par injection unique pour les patientes subissant un accouchement par césarienne. Toutefois, une étude récente n'a démontré aucun avantage des blocs TAP par cathéter par rapport aux blocs TAP par injection unique chez des patients subissant une néphrectomie pour don d'organe par laparoscopie. ${ }^{9}$ Les techniques par cathéter, y compris l'analgésie péridurale postopératoire, sont utiles dans certaines populations de patients pour lesquels la prise en charge de la douleur sera probablement difficile, comme par exemple chez les patients très tolérants aux opioïdes ou les patients sous traitement d'agonistes/antagonistes des opioïdes.

Le bloc du muscle carré des lombes n'a pas été examiné dans l'étude de Sultan et coll., malgré des résultats prometteurs dans certaines ERC récentes. Une métaanalyse récente a conclu que, en l'absence de MIT après un accouchement par césarienne, un bloc du muscle carré des lombes réduisait significativement la consommation d'opioïdes à $24 \mathrm{~h}$ par rapport aux comparateurs inactifs. ${ }^{10}$ Fondamentalement, le bloc du muscle carré des lombes est une extension du bloc TAP postérieur; l'anesthésique local est déposé dans les plans fasciaux thoraco-lombaires adjacents au muscle du carré des lombes. Lorsqu'on réalise un bloc du muscle carré des lombes, le médicament injecté pourrait diffuser jusqu'à l'espace paravertébral, bloquant ainsi les fibres nerveuses innervant les viscères abdominaux. ${ }^{6,11}$ Une MAR récente comparant un bloc du muscle carré des lombes à un bloc TAP dans une population de patientes subissant un accouchement par césarienne n'a trouvé que deux petites ERC comparant directement les deux approches. Les deux ERC ont conclu que le bloc du muscle carré des lombes était plus efficace pour réduire la consommation de morphine et prolonger le délai jusqu'à la première requête analgésique. Toutefois, en combinant les estimations directes et indirectes, il n'y avait pas suffisamment de données probantes pour conclure avec certitude qu'une technique était supérieure à l'autre en termes de consommation postopératoire d'opioïdes à $24 \mathrm{~h}$ et de scores de douleur. ${ }^{12}$ En l'absence de MIT, les blocs TAP et du muscle carré des lombes sont tous deux associés à une consommation postopératoire d'opioïdes significativement plus basse par rapport aux témoins.

$\mathrm{Au}$ delà d'une technique régionale en particulier, les avantages d'une analgésie multimodale et des adjuvants à l'analgésie régionale ne devraient pas être oubliés dans un contexte obstétrical. Les qualités bien connues de diminution des opioïdes par une administration programmée d'agents anti-inflammatoires non stéroïdiens (AINS) et d'acétaminophène sont à la base de toute stratégie analgésique. ${ }^{13} \mathrm{La}$ dexaméthasone peut être envisagée dans le cadre d'un régime analgésique multimodal. Il a été démontré qu'une dose intraveineuse 
unique de dexaméthasone réduisait la douleur postopératoire, ${ }^{14}$ prolongeait la durée d'action du bloc nerveux, ${ }^{15}$ et avait un effet antiémétique. Alors que son utilisation est coutumière lors d'une anesthésie générale, malgré ses avantages analgésiques et antiémétiques, la dexaméthasone intraveineuse est bien moins souvent administrée aux patients recevant une anesthésie régionale.

En ce qui concerne l'analgésie après un accouchement par césarienne, l'état actuel des données probantes suggère l'utilisation d'une analgésie multimodale comportant l'administration programmée d'acétaminophène et d'AINS ainsi qu'un bloc TAP postérieur par injection unique échoguidée, en l'absence d'un opiö̈de intrathécal à action prolongée. Ce régime posologique permet d'atteindre un équilibre optimal entre efficacité, faisabilité et innocuité. La dexaméthasone intraveineuse peut être considérée comme un complément sécuritaire et efficace pour prolonger l'analgésie du bloc après un accouchement par césarienne. Une infiltration de l'incision en dose unique n'a pas d'avantage prouvé par rapport à un comparateur inactif. Les techniques d'infiltrations continues de l'incision ne confèreront probablement pas suffisamment de bienfaits analgésiques par rapport aux blocs TAP pour justifier leur utilisation, étant donné les entraves qu'elles constituent au rétablissement des patientes. Des données probantes récentes suggèrent que le bloc du muscle carré des lombes est au moins équivalent au bloc TAP. Étant donné les résultats prometteurs en faveur du bloc du muscle carré des lombes dans des études de comparaison directe, des études supplémentaires comparant le bloc du muscle carré des lombes au bloc TAP sont nécessaires.

Disclosures None.

Funding statement None.

Editorial responsibility This submission was handled by Dr. Philip M. Jones, Associate Editor, Canadian Journal of Anesthesia.

Déclaration Aucune.

Déclaration de financement Aucune.

Responsabilité éditoriale Cet article a été traité par Dr Philip M. Jones, rédacteur adjoint, Journal canadien d'anesthésie.

\section{References}

1. Eisenach JC, Pan PH, Smiley R, Lavand'homme P, Landau $R$, Houle TT. Severity of acute pain after childbirth, but not type of delivery, predicts persistent pain and postpartum depression. Pain 2008; 140: 87-94.

2. Mitchell KD, Smith $C T$, Mechling $C$, Wessel CB, Orebaugh $S$, Lim G. A review of peripheral nerve blocks for cesarean delivery analgesia. Reg Anesth Pain Med 2019; . https://doi.org/10.1136/ rapm-2019-100752.

3. Sultan $P$, Patel SD, Jadin S, Carvalho B, Halpern SH. Transversus abdominis plane block compared with wound infiltration for postoperative analgesia following cesarean delivery: a systematic review and network meta-analysis. Can J Anesth 2020. DOI: https://doi.org/10.1007/s12630-020-01818-x

4. Salanti G, Del Giovane C, Chaimani A, Caldwell DM, Higgins $J P$. Evaluating the quality of evidence from a network metaanalysis. PLoS One 2014; . https://doi.org/10.1371/journal.pone. 0099682.

5. Society for Obstetric Anesthesia and Perinatology (SOAP). Enhanced Recovery After Cesarean (ERAC) Consensus Statement - 2019. Available from URL: https://soap.org//SOAPEnhanced-Recovery-After-Cesarean-Consensus-Statement.pdf (accessed August 2020).

6. Uppal V, Sancheti $S$, Kalagara $H$. Transversus abdominis plane (TAP) and rectus sheath blocks: a technical description and evidence review. Curr Anesthesiol Rep 2019; 9: 479-87.

7. Abdallah $F W$, Laffey $J G$, Halpern $S H$, Brull R. Duration of analgesic effectiveness after the posterior and lateral transversus abdominis plane block techniques for transverse lower abdominal incisions: a meta-analysis. Br J Anaesth 2013; 111: 721-35.

8. Yu HC, Spencer AO, Baghirzada L, Endersby $R$. Reducing the incidence of LAST: don't forget ultrasound guidance. Reg Anesth Pain Med 2019; . https://doi.org/10.1136/rapm-2018000037.

9. Yeap YL, Wolfe JW, Kroepfl E, Fridell J, Powelson JA. Transversus abdominis plane (TAP) block for laparoscopic live donor nephrectomy: continuous catheter infusion provides no additional analgesic benefit over single-injection ropivacaine. Clin Transplant 2020; . https://doi.org/10.1111/ctr.13861.

10. Uppal V, Retter S, Kehoe E, McKeen DM. Quadratus lumborum block for postoperative analgesia: a systematic review and metaanalysis. Can J Anesth 2020; . https://doi.org/10.1007/s12630020-01793-3.

11. Elsharkawy H, El-Boghdadly K, Barrington M. Quadratus lumborum block: anatomical concepts, mechanisms, and techniques. Anesthesiology 2019; 130: 322-35.

12. El-Boghdadly K, Desai N, Halpern S, et al. Quadratus lumborum block vs. transversus abdominis plane block for caesarean delivery: a systematic review and network meta-analysis. Anaesthesia 2020: DOI: 10.1111/anae.15160.

13. Carvalho B, Butwick AJ. Postcesarean delivery analgesia. Best Pract Res Clin Anaesthesiol 2017; 31: 69-79.

14. De Oliveira GS, Jr Almeida MD, Benzon HT, McCarthy RJ. Perioperative single dose systemic dexamethasone for postoperative pain: a meta-analysis of randomized controlled trials. Anesthesiology 2011; 115: 575-88.

15. Hussain N, Van den Langenbergh T, Sermer C, et al. Equivalent analgesic effectiveness between perineural and intravenous dexamethasone as adjuvants for peripheral nerve blockade: a systematic review and meta-analysis. Can J Anesth 2018; 65: 194-206.

Publisher's Note Springer Nature remains neutral with regard to jurisdictional claims in published maps and institutional affiliations. 